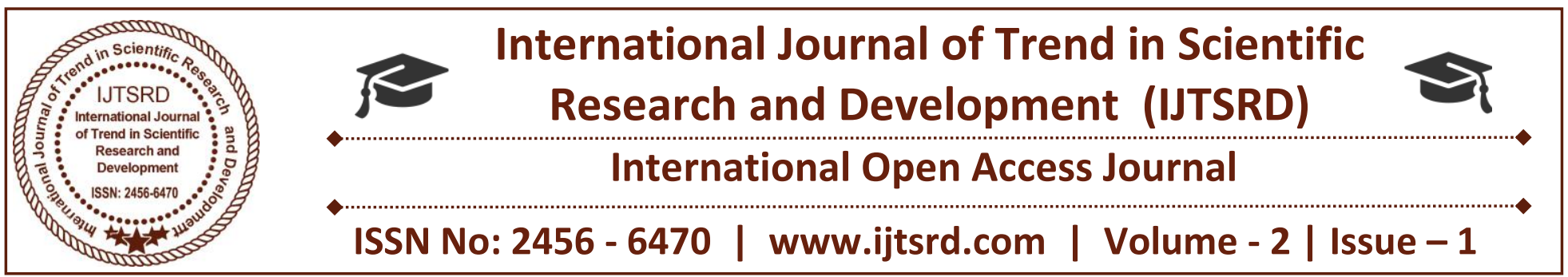

\title{
Image Re-Ranking Modernistic Structure for Web Images
}

\author{
Ms. Sruthi Anand \\ Department of Information Technology, \\ Sri Krishna College of Engineering and \\ Technology,Coimbatore, Tamil Nadu, India \\ R. Vivekalakshmi \\ Department of Information Technology, \\ Sri Krishna College of Engineering and \\ Technology, Coimbatore, Tamil Nadu, India
}

\author{
B. Yaalini \\ Department of Information Technology, \\ Sri Krishna College of Engineering and \\ Technology, Coimbatore, Tamil Nadu, India \\ S. Karthik Raja \\ Department of Information Technology, \\ Sri Krishna College of Engineering and \\ Technology, Coimbatore, Tamil Nadu, India
}

\begin{abstract}
Re-ranking of images is an effective way to improve the results of web-based image search and it has been adopted by the current search engines. When a query keyword is given, a list of images isretrieved first on the basis of textual information given by the user.Nowadays, with user's search intention, web search engine gives a set of relevant as well as irrelevant Images and then the user selects query image from the pool. The remaining Images are reranked based on their visual similarities. The main drawback with current web search engine is that visual similarities of images do not correlate well with Images semantic meaning. We propose a unique web Image re-ranking framework that provides learned Images visual and semantic meaning based on query keywords.
\end{abstract}

Keywords: Semantic meaning of Images, content based-image retrieval, keyword expansion, image reranking

\section{Introduction}

The key aspect of re-ranking of Images is to calculate and identify visual similarities of images. The Internet has become an asset of our everyday life. The query keyword is given as input by the user; a pool of images relevant to the query keyword is fetched by the search engine based on the word-image index file which is stored. The massive and increase in the number of images have shown path for many applications to browse the images on internet. These applications provide easy access to images on web. The problem faced now is that how to model the relevance of images on the internet. Web image search engines use query keywords and depend on the surrounding text to search the images. The user suffers ambiguity of the given query keywords, as it is hard for users to describe the visual content of target images accurately by using keywords only. For example, when "apple" is query keyword, the images that are retrieved belongs to different categories, such as "red apple fruit", "apple MAC book", "apple organisation logo" and "apple iPhones". To solve the ambiguity, content- based image retrieval with relevance is used. The feedback will be incremented by one when the user clicks on that particular image of concern. Using this relevance feedback it will be easy go through the image in which many have chosen to use the same image. When any other user uses the same query keyword to find the image in the web then the highest feedback got by that particular image will appear first on the top. Images are reranked on basis of the learned visual similarities. 


\section{A. Image Retrieval}

Image Retrieval is a concept where the images can be searched and browsed from a large database of images. It is tedious task to access these images from the Web; this becomes difficult to done retrieving of images. Since this is one of the technology, the usage rate and its demand is very high.

In this retrieval system, there have been two different research parts which motivated research. The first one is old information retrieval that follows text oriented information for image retrieval and uses various methods and processes for image ranking and retrieval. The second one is image retrieval system is the field of computer science, which uses different methods and algorithms in order to analyse and index the images, depending on their image-content, such as texture, colour, pixels and so on.

\section{B. Image retrieval based on the content (cb-ir)}

Image retrieval systems based on contents are differentiated into many hierarchies of concepts. At the lowest hierarchy, retrieval of image is on the basis of primary characteristics such as shape, colour of the image and texture. Higher hierarchies explain upper level of semantic spaces features, such as mono objects, types of objects, events and the presentation consequence.

Image retrieving technology on contents based systems has a detailed region of learning, which has acquired very much importance from the past years. Since this technology is used in numerous areas such as pharm medicals, law , crime detection, fashion designing and many more, the characteristic of getting the visual information has become important and the notch area of information technology. Image retrieving was used earlier which was started to retrieve images based on textual approach. This method did not become popular and it had ambiguity associated with this kind of approach. The main problem was the use of annotation of words, which caused difference in observation of images. This is the main reason why the researchers moved to think on CB-IR based systems, where the images are retrieved or got by automation of the derived features.

\section{Literature Review}

Ramachandran (2015) [1] proposed a novel re-ranking of images framework which automatically learns different semantics for query keywords and displays image details in an augmented manner. The images are worked on their related semantic spaces to retrieve their semantic signatures with a one click feedback from the user. Re-ranking of images done with the help of semantic meaning of images in their semantic spaces obtained by keyword. The proposed queryspecific semantic signatures improve both the efficiency and accuracy of re-ranking of images.

Shi Qiu (2014) [2] proposed a novel visual semantic complex network that models the complex structures of a web image collection framework. Multiple fundamental structures of complex networks were studied, which revealed some interesting facts about the VSCN. They not only help us understand the huge web image collection at a macroscopic level, but are also very valuable in practical applications. Two different applications show that making use of information on structure of the VSCN not only improves CBIR, but it also improves the experience of the userin searching and browsing images on web images.

Ambreen Anjum (2013) [3] built a search engine based on visual content that exploits the digital image characteristics to search the database of images and output accurate results. The author proposed an algorithm that searches the images based on its contents and then produces efficient and accurate output of results. The performance of the algorithm is very much better than the previous algorithms.

A unique web Image re-ranking framework is presented that learned Images visual and semantic meaning regarding with numerous query keywords.[2] These visual and semantic meaning of Images extended to visual semantic space of Image to get visual semantic signatures. The modules are

$\begin{array}{ll}\text { - } & \text { Keyword Expansion } \\ \text { - } & \text { Kisual Semantic Signatures } \\ & \text { K-means clustering }\end{array}$

\section{1) Keyword Expansion}

Keyword expansions are estimated based on most relevant word for query keyword that directly along with by automatically way selected by system to exploit both textual as well as visual information. [4]

The keyword ' $\mathrm{k}$ ' is checked in the image database and the image is retrieved based on expanded keyword. 


\section{2) Visual Semantic Signatures}

Using visual semantic signatures different user provide numerous query to search for different result images over the web and then the web based search engine will give return back numerous images that similar to the search query keyword which provided by the user. So, similarity criteria for search criteria could have include in following different form like Meta tags, region, shape and colour distribution in image setFor the given $\mathrm{M}$ classes of reference for query keyword $\mathrm{q}$ and their Images, multi class classifier on visual characteristics of images is formed and the M-dimensional vector which indicates the probabilities of the new image belonging to different reference classes and it calculates the semantic signatures for each image namedI. For retrieve images from web clustering is applied to calculate clustering criteria for query keyword. [4]

\section{3) K-means clustering}

In k-means clustering centroid vector is computed for each cluster. For minimizing sum of cluster distance centroid vector is used. The Euclidean distance used for region-based on Image semantic meaning that represented as set of weighted clusters. Finally, centroid for each cluster is formed that recognize as discrete distribution. [5]

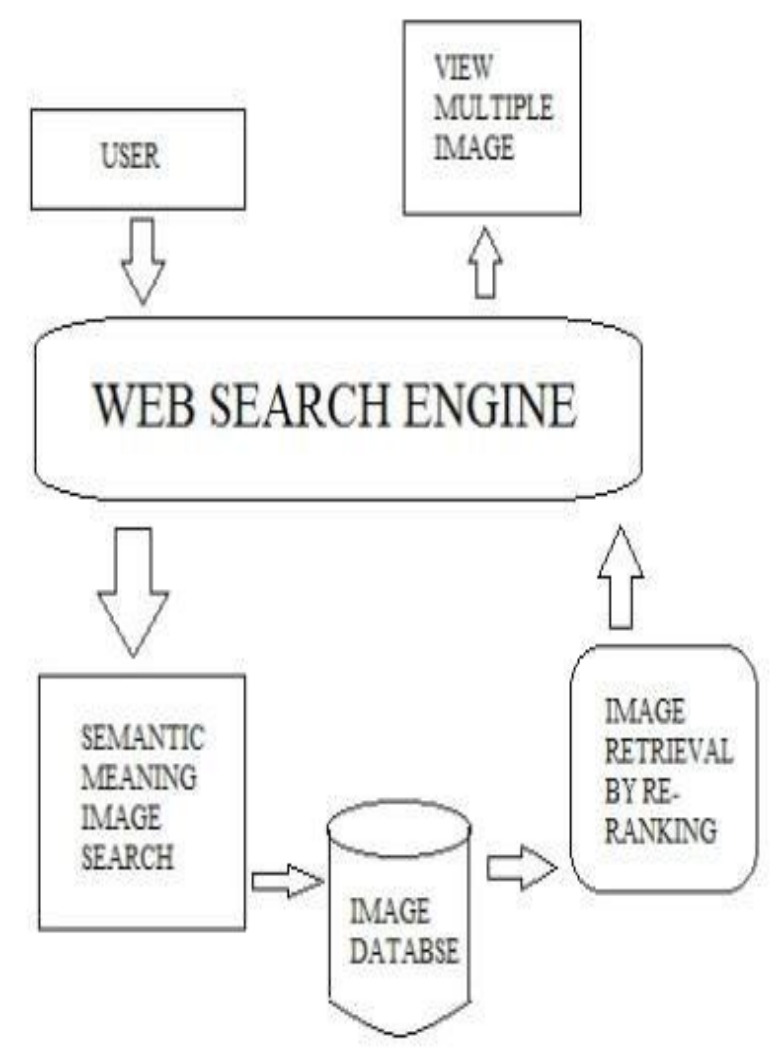

Fig 1: Overall Architecture [4]

\section{RESEARCH METHODOLOGY}

In present search engine, a different number of users enter keyword as queries and then based on surrounding text numerous images are extricated to the user. Internet-scale Web Search motors for the most part exploit and take query keyword as questions i.e. content to inquiry pictures of target pictures just utilizing query keyword.In this method, the visual similarities of images do not correlate and coincide well with the image's semantic meaning and semantic space which is the main drawback with the current existing web image search engine.Key issue to calculate images visual and semantic features are not solved .Catch client expectation from a single click query keyword is not present.

We propose novel structure for web search engine for web images recompilation that recognizes different examples. In the existing system, when any user gives query keyword input on the web search engine as say "fruit", then related semantic spaces of the query is lessened. In the proposed concept, the need is to memorize the visual and textual characteristics of images. Then the context meaning of image is designed so that the original meaning of the requested image is formed to get the semantic meaning for each image. In the online phase, these images are repositioned based on priority by comparing their original meaning obtained from learning the image's visual similarities of the input query keyword. The semantic occurrence between Images is detected by learning the visual correlation between Images and integrates the correlation by giving the similarity of search Image. It is also possible for numerous users to use this framework for online search of images from web Image search engine and based on the search query re-ranking of Images is posed to the user. The purpose of this work is to automatically discover and model the visual and semantic structures of web image pools, study their properties and demonstrate the use of such structures through applications. 


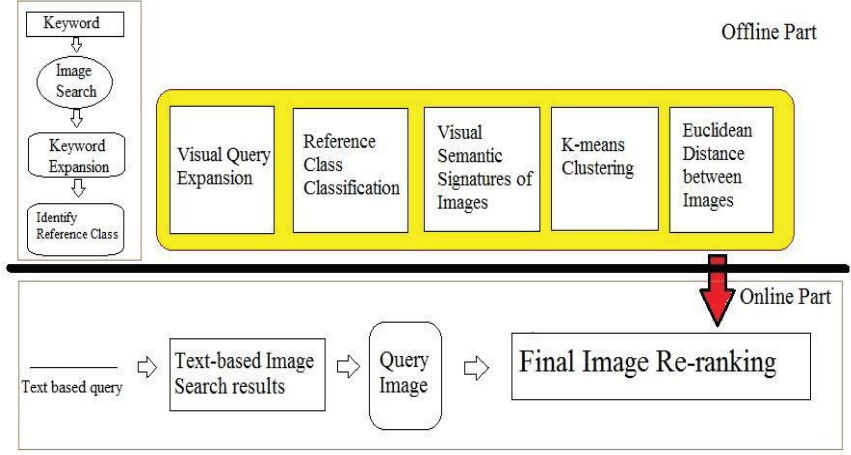

Fig. 2 Flow diagram of proposed concept [4]

The query keyword is given as input by the user. Based on the content, the images are searched from the database. The keyword is expanded and it is referred with class classification. With the help of $\mathrm{K}$ means clustering algorithm centroid is formed for the images Euclidean distance between images are calculated. Images with smaller distance are given to the users. The images are retrieved and other images are re-ranked based on Euclidean distance.

\section{Conclusion}

We concluded that the design of innovative Image re-ranking modernistic structure for web Image reranking technique. It also provides efficiency and accuracy for the re-ranked Image. In our framework particular query keyword visual semantic signature to get more modernized re-ranking of Image. In our setup we accomplish $20-30 \%$ superior result on reranking technique.

There is also a probability to capture keyword expansion that is useld to denote the classes of reference that accommodate data about data nothing but metadata and $\log$ that is present in the text-based and visual trace of the image. In future instead of giving keyword as input, image can be given. That image's semantic meaning will be captured and images retrieved with enhanced accuracy.

\section{REFERENCES}

1. A Ramachandran, M Sai Kumar, Dr. C. Nalini, "Learning Image Re-Rank: Query-Dependent Image Re-Ranking Using Semantic Signature", International Journal of Innovative Research in Science, Engineering and Technology, Vol. 4, March 2015.

2. Xiao gang Wang, Member, IEEE, Shi Qiu, Ke Liu, and Xiaoou Tang, Fellow, "Web Image ReRanking Using Query-Specific Semantic Signatures, " IEEE transactions on pattern analysis and machine intelligence, vol. 36, no. 4, April 2014.

3. Ambreen Anjum ,Muhammad Nabeel Aslam, Rehana Sharif,"The Design of a Semantic Search Engine based onVisual Content", International Journal of Advanced Research in Computer Science and Electronics Engineering, Volume 2, April 2013.

4. Mr.Sandesh Keshav Pawaskar, Mr. S. B. Chaudhari, "Web Image Search Engine Using Semantic of Images's Meaning for Achieving Accuracy," International Institute of Information Technology ,Pune,2016.

5. J.Cai, Z.Zha, W.Zhou, and Q.Tian,"AttritubeAssisted re-ranking for web Image Retrievel", in Proc 20th ACM Int conf.Multimedia 2012.

6. X. Tang, K. Liu, J. Cui, F. Wen, and X. Wang. Intent search: "Capturing user intention for oneclick internet image search", TPAMI, 2012.

7. J. Cui, F.Wen, and X. Tang. "Real time Google and live image search re-ranking" In Proc. ACM MM, 2008.

8. (2016) The IEEE website. [Online]. Available: http://www.ieee.org/

9. Live Google Image Search. Follow http://Image.google.com.

10. Microsoft Live Image Search follow http://www.live.com 